\title{
Quantitative evaluation of computed tomography findings in patients with pulmonary embolism: the link between D-Dimer level and thrombus volume
}

\author{
Hadi Sasani ${ }^{1 *} \odot$, Levent Cem Mutlu²
}

\begin{abstract}
SUMMARY
OBJECTIVE: To investigate the correlation of D-dimer levels and computed tomography properties of pulmonary embolism. METHODS: A total of 58 treated patients with diagnosis of properties of pulmonary embolism were retrospectively studied. All patients underwent a D-dimer blood test. In computed tomography images, septal angle, interventricular septal thickness, and the diameters of all cardiac chambers and pulmonary arteries were measured. The thrombus volume (load) and density at all pulmonary arteries (main, right, left pulmonary arteries, and segmental arteries) were calculated.

RESULTS: A significant correlation was found between D-dimer and total thrombus volume $(p=0.009, r=0.342)$. Total thrombus volume and total thrombus density were calculated with mean value of $23.40 \pm 60.63 \mathrm{ml}$ and $66.16 \pm 38.48$ hounsfield unit (HU), respectively. Right ventricle/left ventricle ratio showed positive correlation with the $D$-dimer level $(p=0.02)$.

CONCLUSION: Increased D-dimer levels with RV/LV ratio and their correlation with total thrombus volume suggest that it may be a prognostic factor.

KEYWORDS: Thrombosis. D-dimer. Pulmonary embolism. X-Ray computed tomography. Quantitative analysis.
\end{abstract}

\section{INTRODUCTION}

Pulmonary embolus (PE) refers to obstruction of the pulmonary artery or one of its branches by material (e.g. thrombus, tumor, air, or fat) that originated elsewhere in the body. Acute PE is a common and fatal disease whose mortality rates can be as high as 31 to $58 \%$ when shock is present ${ }^{1}$. It increases the pressure of the pulmonary arterial system and right ventricle (RV), resulting in RV dysfunction, which may progress to right heart failure and circulatory collapse $\mathrm{e}^{2,3}$.

Difficulty may occur in diagnosis due to its clinical nonspecific nature and presentation with different clinical situations. Initiation of the treatment just after immediate diagnosis provides a decrease in morbidity and mortality.
Patients can be classified as massive and sub-massive according to their hemodynamic stability. For the confirmation of the probability of PE, D-dimer level is the most commonly used laboratory parameter in routine clinical practice. $\mathrm{D}$-dimer is formed upon breaking down the cross-linked fibrin. In patients with suspected PE, blood D-dimer levels correlate with the probability of having $\mathrm{PE}^{4,5}$.

As an imaging modality, computed tomographic pulmonary angiography (CTPA) is the frontline imaging modality in the patients with suspected acute $\mathrm{PE}^{6,7}$. CTPA is an essential component in commonly used clinical diagnostic algorithms with the highest sensitivity $(83 \%)$ and specificity $(96 \%)^{8}$. PE clot volume can be measured in CTPA setting using a semi-automated algorithm?.

${ }^{1}$ Tekirdag Namik Kemal University, Faculty of Medicine, Department of Radiology - Tekirdag, Turkey.

${ }^{2}$ Tekirdag Namik Kemal University, Faculty of Medicine, Department of Chest Diseases - Tekirdag, Turkey.

*Corresponding author: hsasani@nku.edu.tr

Conflicts of interest: the authors declare there is no conflicts of interest. Funding: none.

Received on August 14, 2020. Accepted on November 23, 2020. 
To the best of our knowledge and the literature, there is no study correlating total thrombus volume (TTV: the thrombus load in the main and both pulmonary arteries including segmental branches) with D-dimer level.

The objective of this article was to investigate the correlation of D-dimer level and CT properties of thrombosis such as TTV and RV/left ventricle (LV) ratio.

\section{METHODS}

\section{Study population}

Between May 2016 and May 2019 in the hospital of the institution, in a total of 58 patients treated with the diagnosis of PE, were studied retrospectively. All patients had D-Dimer blood test. Patients with inadequate quality of CT images or insufficient breathing during CT scanning, patients with chronic diseases (malignancy, cardiac insufficiency or chronic cardiac diseases), and those under 18 years of age were excluded from the study.

Patients between 18-65 years of age with optimum CTPA image quality and breathing, patients clinically and radiologically diagnosed with PE, were enrolled in the study.

The study protocol was approved by the institutional ethics review committees (approval number: 2019.191.10.12).

\section{Computed tomography pulmonary angiography acquisition}

A 128-row multi-detector CT scanner (Aquilion ${ }^{\mathrm{TM}}$ Prime; Canon Medical Systems) was used for CTPA scanning. Field of view (FOV) of the whole chest was scanned from the lung apex to the diaphragm with a single breath-hold.

\section{Computed tomography protocol}

CT acquisition was done by the following parameters: the current of 100-250 mAs modulated by personal body mass index dose; tube voltage of $100-140 \mathrm{kV}$ and collimation of $0.5 \mathrm{~mm}$ $\mathrm{x} 80$, gantry rotation time of $0.35 \mathrm{sec}$, pitch factor of 0.813 , FOV: $20 \times 20 \mathrm{~cm}$, slice thickness of $1 \mathrm{~mm}$, and slice interval of $0.8 \mathrm{~mm}$. For intravenous bolus injection of non-ionic contrast material $\left(350 \mathrm{mg} / 100 \mathrm{~mL}\right.$, Iohexol, Omnipaque ${ }^{\circledR}$; GE Healthcare, Cork, Ireland), a mechanical injector was used at a flow rate of $4.5-5.0 \mathrm{~mL} / \mathrm{sec}$. The automatic bolus-tracking method was used with the ROI (region of interest) positioned at the level of the main pulmonary artery with a pre-defined threshold of $100 \mathrm{HU}$, and a fixed delay of $5 \mathrm{sec}$ was used for data acquisition. Electrocardiogram (ECG)-gating technique was not performed. Patients with good quality of images, without artifacts, and those the largest ventricular diameters were enrolled in the study. Cardiac measurements were made in the phase where the largest ventricle diameters were thought to be in the diastolic phase.

\section{Imaging review}

An eight-year-experienced radiologist assessed the patients for the presence of $\mathrm{PE}$ and analyzed the distribution within the main pulmonary vasculature and segmental arteries bilaterally.

After acquisition, the obtained CTPA images were transferred to Picture archiving and communication system (PACS) (Sectra 7.0, Sectra AB, Linköping, Sweden) and were analyzed at the workstation (Vitrea 2 workstation; Vital Images, Minnetonka, MN, USA).

Interventricular septal angle, thickness, and the diameters of all cardiac chambers in a four-chamber plane $(4 \mathrm{CH})$ (Figure 1$)$; and the diameters of main, right and left pulmonary arteries were measured on the axial CT images (Figure 2). The thrombus volume (load) and density at all pulmonary arteries (main, right, left pulmonary arteries, and segmental arteries) were calculated (Figure 3). Determination of the boundaries of the thrombus (seen as an intraluminal filling defect in CTPA) was made using semi-automated (a pixel-based image segmentation method known as region growing algorithm) and manual

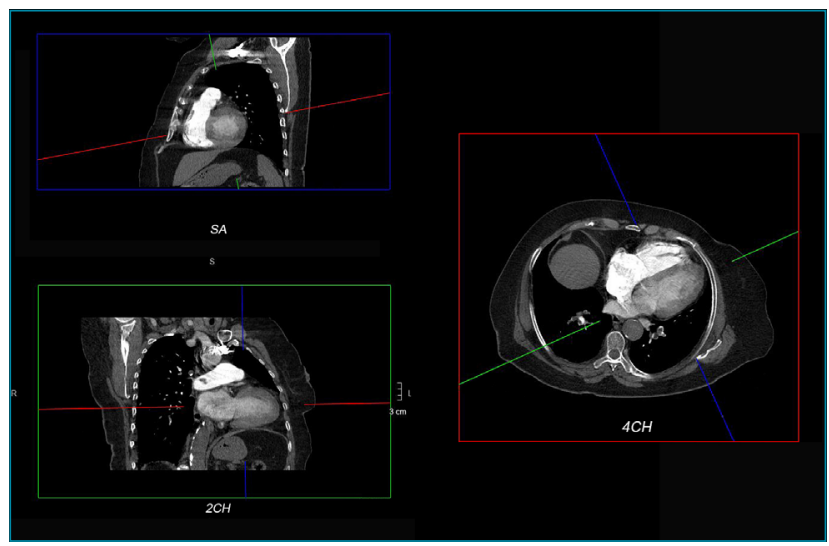

Figure 1. Shows cardiac planes including four-chamber (4CH; including both atria and ventricles), two-chambers $(2 \mathrm{CH}$, one atrium and ventricle) and short axis (SA, just both atria or ventricles).

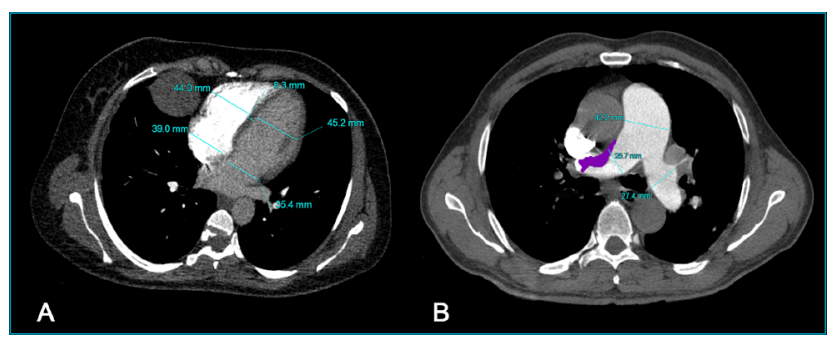

Figure 2. The measurements of cardiac chambers at $4 \mathrm{CH}$ plane (A) and pulmonary arteries $(B)$ are shown. The thrombus area at right pulmonary artery $(B)$. 
drawing tools. The measurements of thrombus volume were categorized into five subgroups including the main pulmonary artery (PA), right $\mathrm{PA}$, left $\mathrm{PA}$, right lung thrombus volume (upper, middle, and lower lobe segmental arteries), and left lung thrombus volume (upper and lower lobe segmental arteries).

The diameter of cardiac chambers was performed between the two endocardia. Interventricular septal angle measurement due to calculation method defined by Tang et al. was done in axial CT images ${ }^{10}$. The angle between the connecting line from the midpoint of the sternum to the thoracic vertebral spinous process and interventricular septum (Figure 4).

\section{Laboratory tests}

In all patients, complete blood count (hemoglobin, hematocrit, neutrophil, leukocyte, and platelet), C-reactive protein, and D-dimer level were analyzed.

\section{Statistical analysis}

All data were analyzed using a statistical package program (SPSS version 17.0; SPSS, Inc., Chicago, IL, USA). The variables were investigated using visual (histograms and probability plots) and analytical methods to determine whether they are normally or not normally distributed. Investigating the associations between non-normally distributed and/or ordinal variables, the correlation coefficients and their significance were calculated using the Spearman test. A 5\% type-1 error level was used to infer statistical significance.

\section{RESULTS}

In a total of 58 cases, 22 were males $(37.9 \%)$ and 36 females $(62.1 \%)$, with a mean age of 60.14 years (minimum 20 years, maximum 93 years).

There was a significant correlation between $\mathrm{D}$-dimer and TTV in the Pearson correlation test $(\mathrm{p}=0.009, \mathrm{r}=0.342)$. $\mathrm{D}$-dimer level was ranging between 0.16 and 22.94 (mean value 6.16 \pm 5.04 ). TTV and total thrombus density (TTD) were calculated with a mean value of $23.40 \pm 60.63 \mathrm{ml}$ (min: $1.46 \mathrm{~mL}$, max: $404.12 \mathrm{ml}$ ) and $66.16 \pm 38.48 \mathrm{HU}$ (min: -67.70 HU, max: $213.50 \mathrm{HU}$ ), respectively (Figure 5). TTD was automatically calculated at the workstation (Vitrea 2 workstation; Vital Images, Minnetonka, MN, USA) when the volume was measured (Figure 1). Demographic properties and CT measurement values of the study population are shown in Table 1 .

The thickness of the interventricular septum was measured with the mean value of $13.46 \pm 11.64 \mathrm{~mm}$.

Interventricular septal angles were measured as $47.88 \pm 22.00$ degrees. There was no statistically significant correlation between angles and TTV.

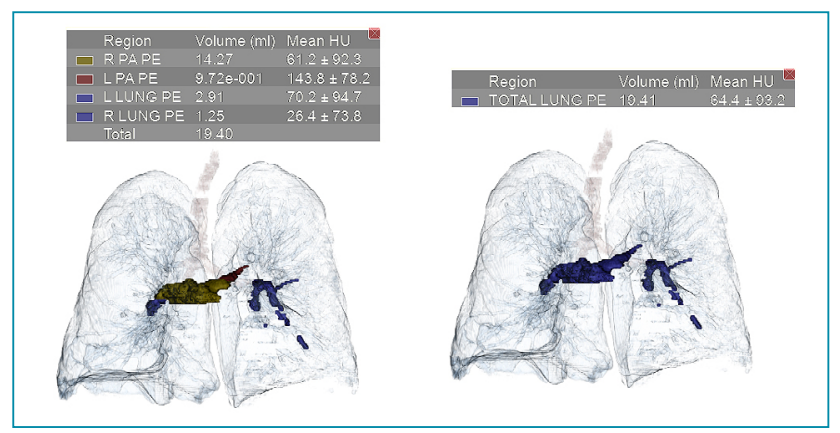

Figure 3. The measurement of thrombus volume (load) and density at main, right, left pulmonary arteries are shown. The thrombus volume of segmental arteries in both lungs is defined as lung PE (A). Total thrombus volume and mean density of total thrombus volume (B).

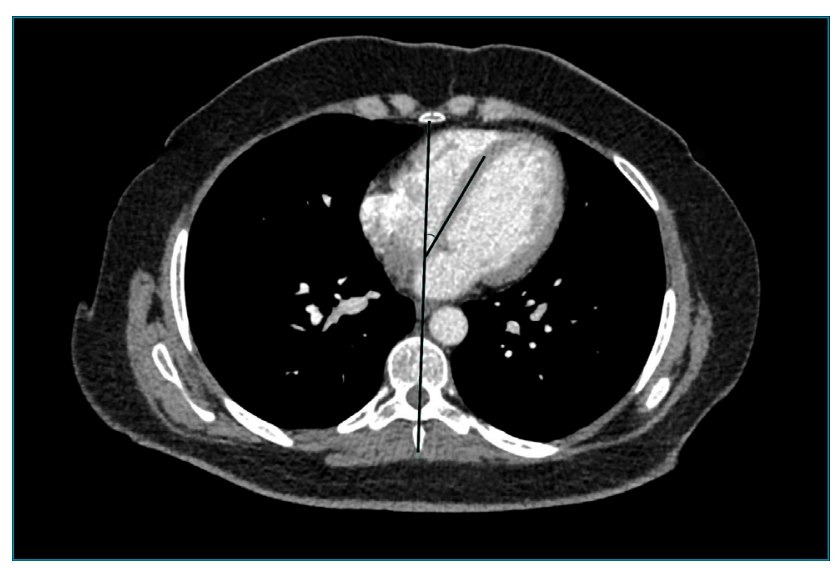

Figure 4. The angle between the connecting line from the midpoint of the sternum to the thoracic vertebral spinous process and interventricular septum.

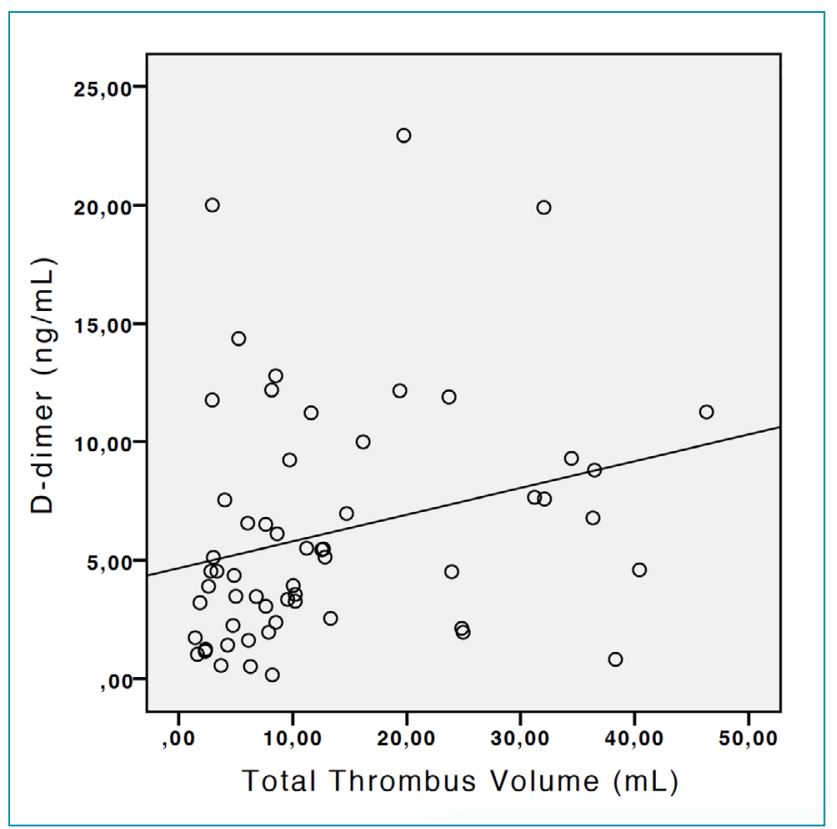

Figure 5. Shows a significant correlation between D-dimer and total thrombus volume $(p=0.009, r=0.342)$. 
In the non-parametric Spearman test, there was a significant correlation between D-dimer and TTV $(\mathrm{p}=0.009)$. A significant correlation was found between TTV and the hematocrit level ( $\mathrm{p}=0.034)$ as well as $\mathrm{C}$-reactive protein (CRP) $(\mathrm{p}=0.044)$.

Interventricular septal angle, and left atrium (LA) and right atrium (RA) diameter showed a significant correlation with TTLV ( $\mathrm{p}=0.023, \mathrm{p}=0.037$, and $\mathrm{p}=0.002$, respectively).

$\mathrm{RV} / \mathrm{LV}$ ratio showed positive correlation with the $\mathrm{D}$-dimer level $(\mathrm{p}=0.02)$.

\section{DISCUSSION}

In PE, although the diagnosis is based on clinical findings, laboratory and imaging findings are crucial. The amount, the location of the thrombus, and the degree of obstruction affect survival $^{11,12}$. In our study, RV/LV ratio showed positive correlation with D-dimer level and a significant correlation was found between D-dimer and TTV.

In some studies, thrombus load has been shown to be predictive of mortality and determination of hemodynamic severity in patients with acute $\mathrm{PE}^{13,14}$. By using measurement parameters (including vascular obstruction, RV/LV ratio, minimum LV diameter, and diameter of the central PA), the thrombus load can be evaluated in the determination of hemodynamic severity ${ }^{14}$. Also, quantitative cardiac CT measurements obtained on axial CT images (RV short axis, LV short axis, and particularly the $\mathrm{RV} / \mathrm{LV}$ short axes ratio) have shown a significant positive (RV short axis, RV/LV diameter ratio) or negative (LV short axis) correlation with the severity of $\mathrm{PE}^{14}$ or with fatal outcomes ${ }^{15,16}$.

Table 1. Demographic properties and CT measurement of the study population.

\begin{tabular}{l|c|c} 
Age & Unit & Value \\
\hline Female & years & $60.14 \pm 17.92$ \\
\hline White blood cell count & $\mathrm{n}(\%)$ & $36(62.1)$ \\
\hline D-dimer & $\mathrm{x} 10^{9} / \mathrm{L}$ & $9.4 \pm 4.4$ \\
\hline CRP & $\mathrm{mg} / \mathrm{dL}$ & $6.11 \pm 5.05$ \\
\hline Platelet & $\times 10^{3} / \mathrm{mL}$ & $251.8 \pm 101.01$ \\
\hline PDW & $\%$ & $15.15 \pm 3.28$ \\
\hline TTV & $\mathrm{mL}$ & $23.40 \pm 60.64$ \\
\hline TTD & $\mathrm{HU}$ & $66.17 \pm 38.49$ \\
\hline Interventricular & $\mathrm{mm}$ & $13.47 \pm 11.65$ \\
septum thickness & degree & $151.88 \pm 22.00$ \\
\hline Septal angle
\end{tabular}

TTV: total thrombus volume, TTD: total thrombus density, CRP: C-reactive protein, PDW: platelet distribution width.
The results of the studies of patients with PE revealed that signs of RV strain at CTPA (RV/LV diameter ratio $>1$, leftward septal bowing) had a sensitivity of $78-92 \%$, specificity of $100 \%$, and positive predictive value of $100 \%$ comparing to echocardiographic findings for the detection of RV dysfunction. Other studies predicted a severe PE when the RV/ $\mathrm{LV}$ diameter ratio was more than $1.5^{14-16}$. In the current study, the diameter ratio of RV/LV in 29 of 58 patients (50\%) with mean value was calculated as $1.17 \pm 0.48$. RV/LV ratio also correlated with D-dimer level. Therefore, increased D-dimer level may indicate an increase in right ventricular tension.

An RV/LV diameter ratio calculation on $4 \mathrm{CH}$ CT images greater than 0.9 has been shown to have association with a sensitivity of $83 \%$ and specificity of $49 \%$ for predicting the occurrence of adverse clinical events (for example 30-day mortality or the need for cardiopulmonary resuscitation, mechanical ventilation, vasopressors, thrombolysis, or embolectomy ${ }^{17}$. Schoepf et al. reported a higher mortality rate in patients with increased RV/LV diameter ratio of more than 0.9 compared to not increased or equal to 0.9 , calculated in $\mathrm{PE}$ patients on $4 \mathrm{CH}$ images. RV enlargement had a sensitivity, specificity, positive predictive value, and negative predictive value of $78.2,38,15.6$, and $92.3 \%$, respectively, for prediction of 30-day mortality ${ }^{18}$.

Similarly, to our study, Kaufman et al. evaluated the reproducibility of PE clot volume quantification using CTPA in a multicenter setting. They used anonymized CTPA data of 23 patients acquired from 23 scanners of 18 imaging centers. Two independent analysts measured PE volumes using a semi-automated region growing algorithm. TTV was calculated per patient as the primary endpoint and individual thrombus volume (ITV), Qanadli score, and modified Qanadli score per patient as secondary endpoints. Inter- and intra-observer reproducibility were evaluated using intra-class correlation coefficient (ICC) and Bland- Altman analysis. As results, clot volumes ranged from 0.0041-47.34 mL (mean \pm SD, $5.93 \pm 10.15 \mathrm{~mL}$ ), found by Analyst 1 . On the second read, analyst 1 found the same number and distribution of emboli with a range of volumes for read 2 from $0.0041-45.52 \mathrm{~mL}$ (mean \pm SD, $5.42 \pm 9.53 \mathrm{~mL}$ ). For Analyst 2, thrombus volumes ranged from $0.00459-46.29 \mathrm{~mL}$ (mean $\pm \mathrm{SD}, 5.91 \pm 10.06 \mathrm{~mL}$ ). Inter- and intra-observer variability measurements indicated excellent reproducibility of the semi-automated approach for quantifying PE volume load. ICC for all endpoints was greater than 0.95 for inter- and intra-observer analysis. No significant biases were indicated by Bland-Altman. They concluded that, the semi-automated region growing algorithm for quantifying PE was a suitable method for image analysis in multicentered clinical trials and was reproducible using data from multiple scanners 9 . By the methodology used in the study, 
they mentioned that the changes in absolute thrombus volume greater than about $2 \mathrm{~mL}$ or $5 \%$ regardless of the starting size of the clots, should be pick up. This could be helpful to estimate sample size requirements for clinical trials using clot burden quantification as an endpoint in PE treatment studies. In the present study, the mean value of TTV was measured as $23.40 \pm 60.63 \mathrm{~mL}$.

The current study was designed as a retrospective study and the patients enrolled were those with acute onset PE. Measurements were only performed on CTPA images statically, no dynamic investigation method was used in the current study such as wall motion abnormality and ejection fraction calculation in ECG or pulmonary wedge pressure at pulmonary catheter angiography. The acquisitions were not done with ECG-gating method.

D-dimer becomes active after $24-48$ hours and then decreases. It may decrease after the patient applies to the hospital; thus, a low D-dimer level might have been analyzed. In this retrospective study, although TTV correlates with D-dimer level, larger prospective studies are needed.

There are some limitations in the in the current study. Firstly, the only diametric measurements of the cardiac chambers were performed in $4 \mathrm{CH}$ plane and the ratio of $\mathrm{RV} / \mathrm{LV}$ was calculated. Measurements of the thrombus volume and determination of its borders were made in contrast enhanced images using semi-automated method and manual drawing option; the thrombus volume in the right, left, and total lungs were calculated, though not separately in segmental branches by lobe. Therefore, the measured thrombus could show variations in density. Secondly, due to the retrospective design nature of the study in the emergency condition, the acquisitions were not performed by ECG-gating method and the cardiac measurements were done at a single cardiac phase. The images with the largest ventricle diameter at diastolic phase were selected for the evaluation. However, it could be better to do it with ECG-triggering technique.

\section{CONCLUSIONS}

Many studies showed thrombus volume to not be a significant determinant of outcomes; rather, the right heart strain is the best predictor. On the other hand, the current study showed TTV and RV/LV ratio had a positive correlation with increasing D-dimer level. Therefore, increased D-dimer levels with $\mathrm{RV} / \mathrm{LV}$ ratio and their correlation with TTV suggest that it may be a prognostic factor, but larger prospective studies are needed to reveal this more clearly.

\section{AUTHORS" CONTRIBUTIONS}

HS: Conceptualization, Data Curation, Writing - Original Draft, Writing - Review \& Editing. LCM: Conceptualization, Formal Analysis, Writing - Review \& Editing.

\section{REFERENCES}

1. Wood KE. The presence of shock defines the threshold to initiate thrombolytic therapy in patients with pulmonary embolism. Intensive Care Med. 2002;28(11):1537-46. https:// doi.org/10.1007/s00134-002-1486-0

2. Kasper W, Konstantinides S, Geibel A, Olschewski M, Heinrich $\mathrm{F}$, Grosser KD, et al. Management strategies and determinants of outcome in acute major pulmonary embolism: results of a multicenter registry. J Am Coll Cardiol. 1997;30(5):1165-71. https://doi.org/10.1016/s0735-1097(97)00319-7

3. Ribeiro $A$, Lindmarker $P$, Juhlin-Dannfelt $A$, Johnsson $H$, Jorfeldt L. Echocardiography Doppler in pulmonary embolism: right ventricular dysfunction as a predictor of mortality rate. Am Heart J. 1997;134(3):479-87. https://doi.org/10.1016/s00028703(97)70085-1

4. Linkins LA, Bates SM, Ginsberg JS, Kearon C. Use of different $\mathrm{D}$-dimer levels to exclude venous thromboembolism depending on clinical pretest probability. J Thromb Haemost. 2004;2(8):1256-60. https://doi.org/10.1111/j.15387836.2004.00824.x

5. Kohn MA, Klok FA, van Es N. D-dimer interval likelihood ratios for pulmonary embolism. Acad Emerg Med. 2017;24(7):832-7. https://doi.org/10.1111/acem.13191
6. Schoepf UJ, Costello P. CT angiography for diagnosis of pulmonary embolism: state of the art. Radiology. 2004;230(2):329-37. https://doi.org/10.1148/radiol.2302021489

7. Goldhaber SZ, Bounameaux H. Pulmonary embolism and deep vein thrombosis. Lancet. 2012;379(9828):1835-46. https:// doi.org/10.1016/S0140-6736(11)61904-1

8. Stein PD, Fowler SE, Goodman LR, Gottschalk A, Hales CA, Hull RD, et al. Multidetector computed tomography for acute pulmonary embolism. N Engl J Med. 2006;354(22):2317-27. https://doi.org/10.1056/NEJMoa052367

9. Kaufman AE, Pruzan AN, Hsu C, Ramachandran S, Jacobi A, Patel I, et al. Reproducibility of thrombus volume quantification in multicenter computed tomography pulmonary angiography studies. World J Radiol. 2018;10(10):124-34. https://doi. org/10.4329/wjr.v10.i10.124

10. Tang Q, Liu M, Ma Z, Guo X, Kuang T, Yang Y. Non-invasive evaluation of hemodynamics in pulmonary hypertension by a Septal angle measured by computed tomography pulmonary angiography: comparison with right-heart catheterization and association with $\mathrm{N}$-terminal pro-B-type natriuretic peptide. Exp Ther Med. 2013;6(6):1350-8. https://doi.org/10.3892/ etm.2013.1324 
11. Qanadli SD, El Hajjam M, Vieillard-Baron A, Joseph T, Mesurolle B, Oliva VL, et al. New CT index to quantify arterial obstruction in pulmonary embolism: comparison with angiographic index and echocardiography. AJR Am J Roentgenol. 2001;176(6):1415-20. https://doi.org/10.2214/ ajr.176.6.1761415

12. Mastora I, Remy-Jardin M, Masson P, Galland E, Delannoy $V$, Bauchart JJ, et al. Severity of acute pulmonary embolism: evaluation of a new spiral CT angiographic score in correlation with echocardiographic data. Eur Radiol. 2003;13(1):29-35. https://doi.org/10.1007/s00330-002-1515-y

13. El-Menyar A, Nabir S, Ahmed N, Asim M, Jabbour G, Al-Thani $\mathrm{H}$. Diagnostic implications of computed tomography pulmonary angiography in patients with pulmonary embolism. Ann Thorac Med. 2016;11(4):269-76. https://doi.org/10.4103/18171737.191868

14. Collomb D, Paramelle PJ, Calaque O, Bosson JL, Vanzetto $G$, Barnoudet D, et al. Severity assessment of acute pulmonary embolism: evaluation using helical CT. Eur Radiol. 2003;13(7):1508-14. https://doi.org/10.1007/ s00330-002-1804-5
15. van der Meer RW, Pattynama PM, van Strijen MJ, van den BergHuijsmans AA, Hartmann IJ, Putter $\mathrm{H}$, et al. Right ventricular dysfunction and pulmonary obstruction index at helical CT: prediction of clinical outcome during 3-month follow-up in patients with acute pulmonary embolism. Radiology. 2005;235(3):798803. https://doi.org/10.1148/radiol.2353040593

16. Ghaye B, Ghuysen A, Willems V, Lambermont B, Gerard P, $D^{\prime}$ Orio $V$, et al. Severe pulmonary embolism:pulmonary artery clot load scores and cardiovascular parameters as predictors of mortality. Radiology. 2006;239(3):884-91. https://doi. org/10.1148/radiol.2392050075

17. Quiroz R, Kucher N, Schoepf UJ, Kipfmueller F, Solomon SD, Costello P, et al. Right ventricular enlargement on chest computed tomography: prognostic role in acute pulmonary embolism. Circulation. 2004;109(20):2401-4. https://doi. org/10.1161/01.CIR.0000129302.90476.BC

18. Schoepf UJ, Kucher N, Kipfmueller F, Quiroz R, Costello P, Goldhaber SZ. Right ventricular enlargement on chest computed tomography: a predictor of early death in acute pulmonary embolism. Circulation. 2004;110(20):3276-80. https://doi. org/10.1161/01.CIR.0000147612.59751.4C

\section{ERRATUM}

https://doi.org/10.1590/1806-9282.67.02.20200539ERRATUM

In the manuscript "Quantitative evaluation of computed tomography findings in patients with pulmonary embolism: the link between D-Dimer level and thrombus volume”, DOI: 10.1590/1806-9282.67.02.20200539, published in the Rev Assoc Med Bras. 2021;67(2):218-223.

\section{Page 219, Methods, Study population, second paragraph:}

\section{Where it reads:}

Patients between 18-65 years of age with optimum CTPA image quality and breathing, patients clinically and radiologically diagnosed with PE, were enrolled in the study.

\section{It should read:}

Patients aged 18 and over with optimum CTPA image quality and breathing, patients clinically and radiologically diagnosed with PE, were enrolled in the study. 